\title{
ELEMENTOS PARA EL DISEÑO DE PLANES DE EVALUACIÓN DE PROGRAMAS DE TELEFORMACIÓN EN LA EMPRESA
}

(Elements for evaluation plans design about e-learning programs in the company area)

por

$\underline{\text { Article record }}$

$\underline{\text { About authors }}$

Genoveva Ramos (Genoveva.Ramos@uv.es)

Ficha del artículo

HTML format

$\underline{\text { Sobre los autores }}$

Formato HTML

Abstract
From a broad research framework, this paper's objec-
tive is to reflect on different ways to develop E-learning
evaluation plans for companies whose employees are in
continuous training. Two empirical studies are pre-
sented that provide substantive references for identifying
the standards, indicators and variables that can be con-
sidered for this type of evaluation plan. The results of
different kinds of analyses have resulted in a guideline
of standards usable in an Evaluation Protocol, and an
outline to facilitate the planning and development of
these kinds of evaluations.
Keywords
E-learning, E-learning Evaluation Plans, E-learning Pro-
grams design.

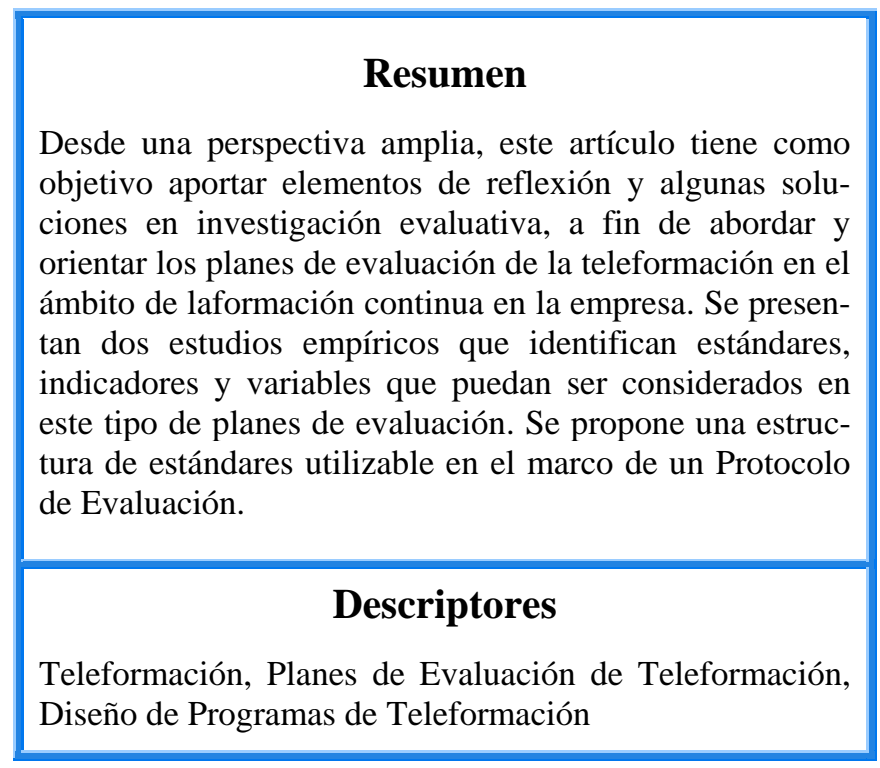

embargo, si que hay un acuerdo común en entender que la evaluación es una de las actividades más necesarias enla planificación, diseño y gestión de la formación en las instituciones educativas y formativas, tanto en el ámbito formal como en el no formal (Jornet 
et al. 2000; Jiménez, 1999; Martínez Mut, 2003).

Desde nuestro punto de vista, es durante el diseño de un programa de formación donde la evaluación ofrece la máxima utilidad, puesto que permite comprobar la eficacia, eficiencia y funcionalidad con el objetivo de identificar los elementos a mejorar $\mathrm{y}$, en definitiva, optimizar el programa de formación. La integración de la evaluación y la planificación como dos dimensiones de la gestión de la formación la convierte en una "función preventiva”, ya que por evaluación no sólo deberemos entender la comprobación del logro de los objetivos, con todas las funciones y objetivos que le podamos asignar, sino también la garantía del cumplimiento de los requisitos o criterios de calidad definidos para cada momento del programa (Martínez Mut, 2003).

Desde esta perspectiva, hemos ido observando distintos modelos, sistemas y planes de evaluación que se han ido desarrollando en el entorno de la teleformación y hemos comprobado que resultan tentativos y parciales, caracterizándose - en la mayor parte de las ocasiones- por centrarse en los elementos tecnológicos. Esto es, la referencia a la tecnología se considera en los planes de evaluación por su atención a la calidad del medio, del instrumento, y no a su integración pedagógica o al valor que ésta tiene dentro del programa de formación. Es decir, el énfasis puede variar hacia elementos organizacionales o hacia componentes específicos, como es el caso de los materiales de formación, dejando la valoración del contexto en el que se desarrolla el programa de formación sin atender o reflejada en pocos elementos dentro de los planes de evaluación.

En este trabajo se presentan distintos núcleos de análisis que entendemos pueden constituir el punto inicial desde el que abordar el desarrollo de una línea de investigación, que conduzca a conocimientos metodológicos sólidos y que permita plantear las bases para desarrollar un modelo o sistema de evaluación de programas de teleformación centrado en todos los elementos que integran esta forma de concebir la formación.

\section{PLANTEAMIENTO DE LA IN- VESTIGACIÓN}

Esta investigación se organiza en torno a una experiencia evaluativa real ${ }^{[1]}$ desarrollada en 1999 por el Departamento MIDE ${ }^{[2]}$ Universitat de València-, en colaboración con Studio 2000 (Empresa dedicada a la teleformación), en el marco de las iniciativas ADAPT de la Unión Europea ${ }^{[3]}$.

Desde el punto de vista de la investigación evaluativa, la información recabada a través de dicho Proyecto/ Experiencia permitió desarrollar estudios dirigidos a la definición de elementos de diseño y modelización de planes de evaluación de programas de teleformación.

\section{OBJETIVO DE LA INVESTIGA- CIÓN}

El objetivo principal del presente trabajo se sitúa en la aportación de información que facilite la construcción de conocimiento en investigación evaluativa para abordar los planes de evaluación de la teleformación en la empresa.

\section{ANÁLISIS Y RESULTADOS}

Con la idea de alcanzar el objetivo planteado en la investigación se llevaron a cabo dos estudios empíricos, en los cuales se desarrollaron objetivos específicos que nos permitieron conseguir lo propuesto y que a continuación pasamos a comentar de manera muy resumida.
4.1 Estudio Empírico I. Diseño y análisis de viabilidad de estándares para la eva- luación de la eficiencia/ funcionalidad del diseño de programas de teleformación. 


\subsubsection{Objetivos del Estudio I}

El objetivo de este primer estudio es observar la eficiencia/ funcionalidad didáctica que los recursos tecnológicos tienen sobre distintos programas de formación continua y analizar cómo funcionan para establecer criterios de evaluación, identificando qué recursos son fundamentales en el diseño de programas de teleformación.

De ahí que aportemos un sistema que facilite la evaluación de los diseños de programas de teleformación teniendo en cuenta la adecuación de la tecnología utilizada para el mejor ajuste metodológico- didáctico de los programas.

\subsubsection{Muestra Estudio I}

Este estudio de investigación evaluativa está basado en el juicio de expertos, por lo que nos encontramos con una muestra formada por un colectivo de siete jueces. Dichos jueces eran especialistas en formación, con amplia experiencia como supervisores del diseño y desarrollo del tipo de curso evaluado (tres expertos en Formación Ocupacional y Continua; y cuatro gestores y técnicos de Formación en grandes Entidades de Formación Ocupacional y Continua).

\subsubsection{Variables Estudio I}

La teoría y la práctica de los programas de formación que son objeto de estudio ${ }^{[4]}$ - en total se partía de 366 programas de formación referidos a 16 Familias Profesionales ${ }^{[5]}$ . A los jueces se les aportó la siguiente descripción de cada programa de formación en forma de cuadernillo de referencia establecidos por el INEM - ver tabla 1-:

TABLA 1. Descripción de los elementos de referencia de cada Programa de Formación aportada a los Jueces.

\begin{tabular}{|c|c|}
\hline \multicolumn{2}{|r|}{ DESCRIPCIÓN DE VARIABLES } \\
\hline $\begin{array}{l}\text { A) DATOS } \\
\text { GENERALES } \\
\text { REFERENTES } \\
\text { AL CURSO }\end{array}$ & $\begin{array}{l}\text { Familia Profesional } \\
\text { Denominación del curso } \\
\text { Objetivo general } \\
\text { Requisitos del profesorado: nivel académico, experiencia profesional, nivel pedagógico. } \\
\text { Requisitos de acceso del alumno: Nivel de conocimientos generales, nivel profesional o } \\
\text { técnico, condiciones físicas. } \\
\text { Número de alumnos } \\
\text { Relación secuencial de módulos o grupos de materias integrantes del curso } \\
\text { Duración en horas del tiempo docente } \\
\text { Instalaciones: aulas de clases teóricas, instalaciones prácticas. } \\
\text { Equipo y material: herramientas utillaje, material de consumo, materiales didácticos, ele- } \\
\text { mentos de protección. } \\
\text { Inclusión de Nuevas Tecnologías }\end{array}$ \\
\hline $\begin{array}{l}\text { B) DATOS } \\
\text { ESPECÍFICOS } \\
\text { DE CADA } \\
\text { MÓDULO } \\
\text { INTEGRANTE } \\
\text { DEL CURSO }\end{array}$ & $\begin{array}{l}\text { Denominación del módulo } \\
\text { Objetivo del módulo } \\
\text { Duración del tiempo docente del módulo } \\
\text { Contenido formativo del módulo: prácticas, conocimientos profesionales. }\end{array}$ \\
\hline
\end{tabular}

\subsubsection{Indicadores Estudio I}

Los indicadores establecidos - ver tabla 2se sustentaron sobre una escala de valoración de 0 a 4, donde 0 indicaba la no teleformabilidad y 4 cuando las posibilidades de teleformación eran muy amplias ${ }^{[6]}$. 
TABLA 2. Relación de indicadores tenidos en cuenta en el estudio de Estándares

\begin{tabular}{|c|c|}
\hline \multicolumn{2}{|c|}{ DESCRIPCIÓN DE INDICADORES } \\
\hline $\begin{array}{l}\text { 1. METODOLOGÍA DIDÁCTICA QUE } \\
\text { PERMITE DESARROLLAR CADA RECUR- } \\
\text { SO TECNOLÓGICO: } \\
\text { PÁGINAS HTML } \\
\text { OTROS TIPOS DE PÁGINAS (JAVA, PERL } \\
\text { Y SCRIPT) } \\
\text { CORREO ELECTRÓNICO } \\
\text { GRUPOS DE DISCUSIÓN } \\
\text { RECURSOS CLIENTE/ SERVIDOR } \\
\text { CHAT } \\
\text { VIDEOCONFERENCIA }\end{array}$ & $\begin{array}{l}\text { Se hace necesario conocer la aplicación y su repercusión formativa, to- } \\
\text { mando como criterio a evaluar a cada uno de los recursos tecnológicos, } \\
\text { diferenciando entre recursos de comunicación sincronía (Chat y Video- } \\
\text { conferencia) y recursos de comunicación asíncrona (Páginas HTML, } \\
\text { Otros tipos de Páginas, Correo Electrónico, Grupos de Discusión y Clien- } \\
\text { te/ Servidor). }\end{array}$ \\
\hline $\begin{array}{l}\text { 2. TIPO DE CONTENIDO DEL PROGRA- } \\
\text { MA: MÓDULO TEÓRICO Y MÓDULO } \\
\text { PRÁCTICO }\end{array}$ & $\begin{array}{l}\text { El tipo de contenidos de un Programa puede condicionar el grado de } \\
\text { teleformabilidad de una Ocupación, teniendo en cuenta los módulos } \\
\text { formativos y su duración. Como criterios a evaluar, se tendrán en cuenta } \\
\text { a los contenidos teóricos y prácticos, además de que: } \\
\text { - Se da una situación clara de no teleformabilidad en aquellos casos en } \\
\text { los que no se pueda impartir el programa nada más que de manera pre- } \\
\text { sencial. } \\
\text { - En los casos en que sea susceptible de una formación a distancia se } \\
\text { tratará de valorar si en el desarrollo de los contenidos del programa (teó- } \\
\text { ricos y prácticos) es necesaria y/o produce un mayor beneficio la intro- } \\
\text { ducción de tecnologías para la teleformación. }\end{array}$ \\
\hline $\begin{array}{l}\text { 3. NIVEL DE COMPETENCIA QUE SE ES- } \\
\text { PERA QUE CONSIGAN LOS ALUMNOS }\end{array}$ & $\begin{array}{l}\text { Identificar si los recursos tecnológicos disponibles permiten cumplir con } \\
\text { un desarrollo de programa de formación que pueda satisfacer los reque- } \\
\text { rimientos de competencia planteados como objetivos del programa. Para } \\
\text { ello, asumimos las categorías del Sistema de Clasificación del Rendi- } \\
\text { miento (del Sistema CDT -Teoría Componencial-) de Merrill (Merrill y } \\
\text { Twitchell, 1994): conocimiento, comprensión, aplicación/ utilización. A } \\
\text { partir de ellas podemos disponer de una graduación bastante simple y } \\
\text { representativa de los niveles de competencia genéricos en la cual situar el } \\
\text { nivel de logro alcanzado por los alumnos. Graduación que establecimos } \\
\text { como una dimensión inversa del grado de teleformabilidad; de forma que } \\
\text { los programas más susceptibles de aplicar este tipo de recursos serían } \\
\text { aquellos en los que el nivel de competencia que se esperaba conseguir } \\
\text { fuera menor. }\end{array}$ \\
\hline $\begin{array}{l}\text { 4.NECESIDAD DE DISPONIBILIDAD DE } \\
\text { LOS DIFERENTES RECURSOS TECNO- } \\
\text { LÓGICOS PARA EL DESARROLLO DE } \\
\text { PROGRAMAS MEDIANTE TELEFORMA- } \\
\text { CIÓN }\end{array}$ & $\begin{array}{l}\text { Con respecto al Contenido Teórico y Práctico de un Programa la necesi- } \\
\text { dad/ beneficio de aplicación de los recursos tecnológicos específicos; y } \\
\text { con respecto al Conjunto de un Programa, la adecuación de la teleforma- } \\
\text { bilidad para lograr el nivel de competencia requerido. }\end{array}$ \\
\hline
\end{tabular}

\subsubsection{Metodología y Análisis Estudio I}

Desde el punto de vista metodológico, este estudio se enmarca en procesos de investigación evaluativa, dirigido a recabar información acerca del funcionamiento de estándares en el diseño y desarrollo de planes de evaluación de programas de teleformación. Se desarrollan cuatro análisis, los cuales entendemos pueden constituir el punto inicial desde el que abordar el objetivo de esta investigación:
Análisis 1. Diseño de indicadores de relación entre recurso tecnolóqico utilizado y la metodoloqía didáctica que requiere. Los recursos tecnológicos, en este estudio, son considerados como criterios internos al concepto de teleformabilidad de un programa de formación siendo necesario conocer su aplicación y su repercusión formativa. Es decir, se específica la metodología didáctica que permite desarrollar la utilización de cada uno de ellos para los distintos contenidos de 
formación y de esta manera se establecen los indicadores necesarios para la determinación de estándares.

Análisis 2. Conqruencia Inter-Jueces como consistencia de indicadores y formulación de estándares. El objetivo de este segundo análisis consistió en valorar la congruencia en si misma, como elemento de referencia acerca del valor del estándar para cada programa de formación y seleccionar los indicadores de síntesis estadística para resumir la información relativa a cada programa. Es decir, a partir de los resultados del análisis Inter-Jueces - a través del coeficiente $W$ de Kendall- avalamos la información dada por dichos jueces a través de la consistencia de sus opiniones y observamos las discrepancias existentes entre ellos. Llegándose a establecer un índice de concordancia para el contenido teórico, el contenido práctico y para el global de cada uno de los programas de formación de las distintas familias profesionales analizadas ${ }^{[7]}$.

\section{Análisis 3. Estructuración de estándares. A} partir de los dos análisis anteriores, con este tercero se pretendió establecer, para las distintas familias profesionales, un estándar basado en la identificación de los recursos utilizados y su aprovechamiento en relación con la metodología didáctica que debe desarrollarse en cada programa de formación. Es decir, tratamos de señalar estándares de referencia para evaluar la eficiencia/ funcio- nalidad didáctica ${ }^{[8]}$ de los diseños de programas de teleformación según las distintas familias profesionales estudiadas, analizando como funcionan los indicadores y detectando cuales utilizar para valorar la teleformabilidad de programas de formación y a partir de ello deducir criterios de calidad de dichos programas. Se realiza un análisis descriptivo basado en Medianas cuyos datos hacen referencia al conjunto de programas de formación obtenidos a partir del análisis de concordancia Inter-Jueces.

Análisis 4. Diseño de un protocolo de aplicación de estándares. Por último, con este análisis hemos tratado de aportar información acerca del sistema de teleformación que facilite la evaluación de los diseños de programas de teleformación, por lo que ofrecemos una estructura de estándares que son utilizables desde el marco de un Protocolo de Actuación para Evaluadores ${ }^{[9]}$ - ver Protocolo adjunto en Anexo -.

\subsubsection{Resultados Estudio I}

Teniendo en cuenta el objetivo de este estudio y, por tanto, de los análisis presentados anteriormente, obtenemos como resultado en primer lugar una estructuración de estándares de referencia para evaluar la eficiencia/ funcionalidad didáctica de los diseños de estos programas según las distintas familias profesionales ${ }^{[10]}$ - ver algunos ejemplos en las tablas 3 y 4 -:

TABLA 3. Criterios de Referencia para la Familia Profesional Administración y Oficinas

\begin{tabular}{|c|c|c|c|}
\hline \multicolumn{2}{|c|}{ MÓDULOS TEÓRICOS } & \multicolumn{2}{|c|}{ MÓDULOS PRÁCTICOS } \\
\hline $\begin{array}{c}\text { RECURSO } \\
\text { TECNOLÓGICO }\end{array}$ & $\begin{array}{c}\text { CRITERIO } \\
\text { DE REFERENCIA }\end{array}$ & $\begin{array}{c}\text { RECURSO } \\
\text { TECNOLÓGICO }\end{array}$ & $\begin{array}{c}\text { CRITERIO } \\
\text { DE REFERENCIA }\end{array}$ \\
\hline Otras Páginas & Fundamental & Videoconferencia & Fundamental \\
\hline Páginas HTML & Fundamental & Cliente/ Servidor & Muy importante \\
\hline Correo Electrónico & Muy importante & Otras Páginas & Muy importante \\
\hline Videoconferencia & Muy importante & Correo Electrónico & Importante \\
\hline Chat & Importante & Chat & Importante \\
\hline Grupos de discusión & Importante & Páginas HTML & Poco importante \\
\hline Cliente/ Servidor & Importante & Grupos de discusión & Poco importante \\
\hline
\end{tabular}


Ramos, G. (2005). Elementos para el diseño de planes de evaluación de programas de teleformación en la empresa. RELIEVE: v. 11, n. 2, p. 3-23. http://www.uv.es/RELIEVE/v11n2/RELIEVEv11n2_5.htm

TABLA 4. Criterios de Referencia en la Familia Profesional Comercio

\begin{tabular}{|c|c|c|c|}
\hline \multicolumn{2}{|c|}{ MÓDULOS TEÓRICOS } & \multicolumn{2}{|c|}{ MÓDULOS PRÁCTICOS } \\
\hline $\begin{array}{c}\text { RECURSO } \\
\text { TECNOLÓGICO }\end{array}$ & $\begin{array}{c}\text { CRITERIO } \\
\text { DE REFERENCIA }\end{array}$ & $\begin{array}{c}\text { RECURSO } \\
\text { TECNOLÓGICO }\end{array}$ & $\begin{array}{c}\text { CRITERIO } \\
\text { DE REFERENCIA }\end{array}$ \\
\hline Páginas HTML & Fundamental & Videoconferencia & Fundamental \\
\hline Otras Páginas & Muy importante & Cliente/ Servidor & Fundamental \\
\hline Videoconferencia & Muy importante & Otras Páginas & Muy importante \\
\hline Correo Electrónico & Muy importante & Chat & Importante \\
\hline Cliente/ Servidor & Importante & Páginas HTML & Importante \\
\hline Grupos de discusión & Importante & Correo Electrónico & Importante \\
\hline Chat & Importante & Grupos de discusión & Poco importante \\
\hline
\end{tabular}

Asimismo, este estudio nos ha permitido conocer el grado de eficiencia/ funcionalidad de cada uno de los recursos tecnológicos en las distintas familias profesionales según el contenido teórico y práctico - ver tablas ejemplos 5 y 6-:

TABLA 5. Medianas obtenidas por Otras Páginas en Teoría y Práctica en todas las Familias Profesionales (Escala de 0 a 1)

\begin{tabular}{|l|c|c|}
\hline \multicolumn{3}{|c|}{ OTRAS PÁGINAS (JAVA, PERL O SCRIPT) } \\
\hline \multicolumn{1}{|c|}{ FAMILIA PROFESIONAL } & TEORÍA & PRÁCTICA \\
\hline ADMINISTRACIÓN Y OFICINAS & 0.93 & 0.79 \\
\hline ARTESANÍA & 0.85 & 0.64 \\
\hline CERÁMICA & 0.48 & 0.04 \\
\hline COMERCIO & 0.78 & 0.76 \\
\hline IDIOMAS & 0.72 & 0.76 \\
\hline INDUSTRIA GRÁFICA & 0.63 & 0.26 \\
\hline INDUSTRIA TEXTIL & 0.79 & 0.50 \\
\hline INFORMÁTICA & 0.92 & 0.90 \\
\hline MANUFACTURAS DIVERSAS & 0.46 & 0.07 \\
\hline MONTAJE E INSTALACIÓN & 0.64 & 0.22 \\
\hline SEGUROS Y FINANZAS & 0.91 & 0.75 \\
\hline SERVICIOS A LA COMUNIDAD Y PERSONALES & 0.64 & 0.23 \\
\hline SERVICIOS A LAS EMPRESAS & 0.94 & 0.87 \\
\hline TÉCNICAS EMPRESARIALES & 0.90 & 0.77 \\
\hline TEXTIL & 0.75 & 0.32 \\
\hline TURISMO Y HOSTELERÍA & 0.62 & 0.40 \\
\hline
\end{tabular}

TABLA 6. Medianas obtenidas por la Videoconferencia en Teoría y Práctica en todas las Familias Profesionales (Escala de 0 a 1)

\begin{tabular}{|l|c|c|}
\hline \multicolumn{3}{|c|}{ VIDEOCONFERENCIA } \\
\hline \multicolumn{1}{|c|}{ FAMILIA PROFESIONAL } & TEORÍA & PRÁCTICA \\
\hline ADMINISTRACIÓN Y OFICINAS & 0.64 & 0.88 \\
\hline ARTESANÍA & 0.78 & 0.73 \\
\hline CERÁMICA & 0.53 & 0.30 \\
\hline COMERCIO & 0.78 & 0.90 \\
\hline IDIOMAS & 0.65 & 1.00 \\
\hline INDUSTRIA GRÁFICA & 0.57 & 0.43 \\
\hline INDUSTRIA TEXTIL & 0.68 & 0.41 \\
\hline INFORMÁTICA & 0.73 & 0.84 \\
\hline MANUFACTURAS DIVERSAS & 0.53 & 0.28 \\
\hline MONTAJE E INSTALACIÓN & 0.64 & 0.43 \\
\hline
\end{tabular}


Ramos, G. (2005). Elementos para el diseño de planes de evaluación de programas de teleformación en la empresa. RELIEVE: v. 11, n. 2, p. 3-23. http://www.uv.es/RELIEVE/v11n2/RELIEVEv11n2_5.htm

\begin{tabular}{|l|l|l|}
\hline SEGUROS Y FINANZAS & 0.66 & 0.95 \\
\hline SERVICIOS A LA COMUNIDAD Y PERSONALES & 0.72 & 0.60 \\
\hline SERVICIOS A LAS EMPRESAS & 0.66 & 0.75 \\
\hline TÉCNICAS EMPRESARIALES & 0.70 & 0.88 \\
\hline TEXTIL & 0.68 & 0.21 \\
\hline TURISMO Y HOSTELERÍA & 0.57 & 0.52 \\
\hline
\end{tabular}

Siguiendo con los resultados obtenidos también se da a conocer la eficiencia/ funcionalidad didáctica de cada recurso tecnoló- gico tanto para los contenidos teóricos $(\mathrm{T})$ como prácticos $(\mathrm{P})$ en todos los programas de formación - ver tabla ejemplo 7 -:

TABLA 7. Medianas de Recursos Tecnológicos para los contenidos Teóricos y Prácticos en los Programas de Formación de Administración y Oficinas (Escala 0-1) ${ }^{[11]}$

\begin{tabular}{|c|c|c|c|c|c|c|c|c|c|c|c|c|c|c|}
\hline \multicolumn{15}{|c|}{ Familia Profesional ADMINISTRACIÓN Y OFICINAS } \\
\hline \multirow{3}{*}{$\begin{array}{l}\text { PROGRAMAS DE } \\
\text { FORMACIÓN }\end{array}$} & \multicolumn{14}{|c|}{ RECURSOS TECNOLÓGICOS } \\
\hline & \multicolumn{2}{|c|}{ Html } & \multicolumn{2}{|c|}{$\begin{array}{c}\text { Otras } \\
\text { Páginas }\end{array}$} & \multicolumn{2}{|c|}{ e-Mail } & \multicolumn{2}{|c|}{ Chat } & \multicolumn{2}{|c|}{$\begin{array}{c}\text { Grupo } \\
\text { Discusión }\end{array}$} & \multicolumn{2}{|c|}{$\begin{array}{l}\text { Video- } \\
\text { Confer. }\end{array}$} & \multicolumn{2}{|c|}{$\begin{array}{l}\text { Cliente/ } \\
\text { Servidor }\end{array}$} \\
\hline & $\mathrm{T}$ & $\mathbf{P}$ & $\mathbf{T}$ & $\mathbf{P}$ & $\mathrm{T}$ & $\mathbf{P}$ & $\mathrm{T}$ & $\mathbf{P}$ & $\mathrm{T}$ & $\mathbf{P}$ & $\mathrm{T}$ & $\mathbf{P}$ & $\mathrm{T}$ & $\mathbf{P}$ \\
\hline $\begin{array}{l}\text { Administración del plan } \\
\text { de cuentas }\end{array}$ & ,83 & ,38 & 1,00 & 1,00 & ,70 & ,69 & ,56 & ,50 & 46 & ,46 & 63 & 1,00 & ,52 & 1,00 \\
\hline $\begin{array}{l}\text { Aplicaciones informáticas } \\
\text { de gestión }\end{array}$ & 85 & 48 & 95 & 95 & 75 & ,75 & 60 & 55 & 46 & 35 & 63 & 1,00 & 47 & 75 \\
\hline Hoja de cálculo & ,80 &, 50 & ,95 & 1,00 & ,75 & ,75 & ,58 & ,50 & ,46 & ,38 & 63 & 88 & ,50 & 1,00 \\
\hline Mecanógrafo & ,68 & ,18 & 80 &, 50 & ,63 & ,23 & 47 &, 18 & ,38 &, 18 & ,60 & ,63 & ,41 & ,55 \\
\hline Proceso de textos & ,88 &, 50 & ,95 & 1,00 & ,79 & ,75 & 63 & ,63 & ,58 & 25 & ,63 & 1,00 &, 50 & 1,00 \\
\hline Secretaria de dirección & ,81 & ,21 & 90 & 42 & ,79 & ,40 & 67 & ,25 & 68 & 38 & 72 & 63 & ,47 &, 50 \\
\hline Secretariado &, 81 & ,41 & ,95 & ,69 & ,75 & ,53 &, 59 & ,50 & ,56 & ,30 & 68 & 1,00 & ,46 & ,75 \\
\hline
\end{tabular}

Por lo que a partir de todo ello y como producto final hemos elaborado una guía de aplicación de estándares donde el evaluador podría anticipar si el programa de formación está bien solucionado desde la teleformación. $\mathrm{O}$ lo que es lo mismo, el evaluador teniendo en cuenta los criterios de referencia puede detectar la eficiencia/ funcionalidad de los programas de teleformación - ver Anexo-. Aquí se ofrece una estructura de estándares que son utilizables desde el marco de un Protocolo de Actuación para Evaluadores. Protocolo que puede ser susceptible de un doble uso: por un lado, para analizar la viabilidad del diseño deseado en teleformación y por otro, para incorporar la evaluación a un programa de teleformación. La estrategia de aplicación del Protocolo requiere que el evaluador analice el diseño, y compruebe - a través de entrevistas con los implicados- la adecuación de sus usos. Es decir, se sustenta sobre la evaluación del diseño de teleformación, considerando su acción. Pero esta propuesta, es inicial y tentativa y debería ser probada en otros marcos en los que se pudiese valorar la homogeneidad de utilización de los estándares a través de diferentes situaciones y por diversos evaluadores como base de fiabilidad y validez del Protocolo - ver Anexo-.

\subsection{Estudio Empírico II. Estudio de Va- lidación de un Plan de Evaluación de Teleformación en la Empresa.}

Para la consecución del segundo estudio de esta investigación, partimos del hecho de que la validación de cualquier sistema de evaluación se basa en una acumulación de evidencias acerca de la adecuación del funcionamiento del mismo, es decir, del conjunto de 
estrategias para dotar de validez al proceso evaluativo (Jornet, Suárez y Pérez Carbonell, 2000; Jornet, Suárez y Perales, 2000; Perales, 2000). Estableciéndose la validez como un aspecto multidimensional, aplicado y aplicable a cualquier estrategia de acercamiento a la realidad, desde la investigación básica, los instrumentos de recogida de información a la investigación aplicada y la evaluación (Swanborn, 1996; Perales, 2000). Desde este punto de vista planteamos el siguiente estudio, no obstante, en el sistema de evaluación desarrollado no se realiza un proceso de validación de todo el plan de evaluación sino de una parte de dicho plan: la toma de decisiones o conclusiones.

\subsubsection{Objetivos del Estudio II}

Con este segundo estudio nos planteamos aportar una estructura global de planificación y desarrollo de un proceso de evaluación de programas de teleformación. Así como, apor- tar una valoración acerca de la validez de la definición de indicadores y la utilización de diversas fuentes de información en dicho proceso de evaluación para la toma de decisiones.

\subsubsection{Muestra Estudio II}

Se trata de un estudio de validación basado en las informaciones procedentes de distintas fuentes (agentes claves).

Tipos de muestras:

- Programas de Formación: 10 tipos de programas de formación, aunque algunos de ellos tuvieron varias ediciones, por lo que en total para este proyecto se evaluaron 15 programas. Todos tenían una duración fija de 20 horas de aplicación, presentaban la misma estructura y compartían plataforma de teleformación desarrollada ex profeso para la experiencia - ver tabla 8-.

Tabla 8. Listado de Acciones Formativas

\begin{tabular}{|l|c|}
\hline \multicolumn{1}{|c|}{$\begin{array}{c}\text { Programas de Formación o } \\
\text { Acciones Formativas }\end{array}$} & Ediciones \\
\hline Ofimática Avanzada & 3 \\
\hline Ofimática Básica & 2 \\
\hline Nóminas y Seguros Sociales & 2 \\
\hline Calidad de Servicio al Cliente & 2 \\
\hline Prevención Riesgos Laborales & 1 \\
\hline Análisis Económico Financiero & 1 \\
\hline Contabilidad & 1 \\
\hline Comercio Exterior & 1 \\
\hline Inglés Comercial & 1 \\
\hline Técnicas de Ventas & $\mathbf{1 5}$ \\
\hline
\end{tabular}

- Agentes y fuentes:

- Profesores, Alumnos y Coordinadores de Centros.

- Equipo técnico también denominado Comité de Expertos 1 o Comité Interno. Se denominó así a la Entidad encargada de llevar a cabo la evaluación del proyecto.

- Comité Externo, también denominado Comité de Expertos 2. Conformado por expertos en Pedagogía, Tecnología Educativa y Formación en Empresa.

\subsubsection{Variables e Indicadores Estudio II}

Al constituir un plan completo de evaluación, se integran variables e indicadores tanto de entrada como de proceso y producto, asumibles en diversas finalidades como eficacia, eficiencia y funcionalidad - ver ejemplo en tabla 10-. Todo ello sustentado en diversos tipos de instrumentos:

- Escala de valoración AMEE-13 (Comité de Expertos). 
- Cuestionarios de evaluación de los programas de formación y Guía de entrevista (Alumnos).

- Autoinformes y Guía de entrevista (Profesores/ Tutores).

- Cuestionarios para Coordinadores de formación (Centros).

\subsubsection{Metodología y Análisis Estudio II}

Este estudio se enmarca en procesos de validación como aproximación de investigación evaluativa orientada a determinar el grado en que un plan de evaluación puede dar respuestas al tipo de interrogantes que están a la base de su puesta en marcha. El énfasis en la necesidad de comprobar la validez y utilidad de los diferentes elementos que forman parte de un plan de evaluación, así como, la atención a las características del objeto de estudio, hace que nos situemos en una aproximación basada en la complementariedad metodológica. No obstante, hemos optado por aportar dos grandes bloques de análisis que globalizan la finalidad última del estudio.

Análisis 1. Análisis de la evaluación como contexto de validación: Justificación del plan de evaluación para programas de teleformación. Cualquier proceso de evaluación debe ser considerado un proceso de investigación, proceso que sigue unos pasos y atiende a unos componentes permanentes en su sentido, aunque cambiantes en su especificación al objeto a evaluar. A partir de este posicionamiento y con el objetivo de validar la estrategia de toma de conclusiones del plan de evaluación basado en la triangulación de información procedentes de distintas fuentes e instrumentos, nos planteamos dichos componentes básicos que la evaluación de programas debe atender (Jornet, Suárez, Belloch, 1998):

¿Qué se evalúa?, ¿Para qué se evalúa?, ¿Quién orienta o encarga la Evaluación?, ¿Quién realiza la Evaluación?, ¿Qué audiencias están implicadas?, ¿Qué información se requiere?, ¿A quién se le demanda información?, ¿Cómo se recoge la información?, ¿Cómo se analiza y sintetiza la información?, ¿Quién tiene derecho de acceso a la información?, ¿Cómo se elaboran los informes de Evaluación?

De esta manera, la definición del Plan de Evaluación desarrollado para esta experiencia nos sirve de basa para proceder a nuestro objetivo último, la validación de las conclusiones o toma de decisiones llevadas a cabo por los agentes implicados en este proceso de evaluación y que se desarrolla en el siguiente análisis.

Análisis 2. Estrategia para la validación de las conclusiones /toma de decisiones del Plan de Evaluación.

Aunque la validación deviene de una acumulación de evidencias, nos limitamos aquí a aportar una evidencia inicial, de carácter global. Una estrategia de carácter cualitativa, dirigida a la validación de las conclusiones o toma de decisiones, tomando como punto de partida la triangulación de las informaciones procedentes de fuentes e instrumentos diferenciados.

\subsubsection{Resultados Estudio II}

Como resultado de estos dos análisis y por consiguiente del segundo estudio, se plantea un sistema de recogida de información a través de la triangulación de fuentes e instrumentos teniendo en cuenta los siguientes elementos- ver tabla 9-: 
TABLA 9. Tabla resumen de variables, dimensiones, unidades de análisis, fuentes e instrumentos que hemos tenido en cuenta en el proceso de validación

\begin{tabular}{|c|c|}
\hline \multicolumn{2}{|r|}{ ELEMENTOS DEL PROCESO DE TRIANGULACIÓN } \\
\hline VARIABLES & $\begin{array}{l}\text { Entrada } \\
\text { Proceso } \\
\text { Producto }\end{array}$ \\
\hline DIMENSIONES & $\begin{array}{l}\text { Incorporación al programa } \\
\text { Conocimientos anteriores acerca del contenido del programa } \\
\text { Participación anterior en programas de formación } \\
\text { Conocimientos previos en la utilización del ordenador } \\
\text { Valoración del visor del alumno } \\
\text { Valoración del visor del profesor } \\
\text { Valoración de la organización global de programa } \\
\text { Valoración de los contenidos del programa de formación } \\
\text { Valoración de los recursos tecnológicos } \\
\text { Valoración de los materiales } \\
\text { Valoración del desarrollo de las sesiones } \\
\text { Valoración del profesor/ tutor } \\
\text { Valoración global del programa de formación } \\
\text { Otros aspectos a valorar } \\
\text { Síntesis de puntos fuertes y débiles de la teleformación según su experiencia. }\end{array}$ \\
\hline $\begin{array}{l}\text { UNIDADES DE } \\
\text { ANÁLISIS }\end{array}$ & $\begin{array}{l}\text { Items de los cuestionarios de alumnos y de coordinadores } \\
\text { Elementos del Protocolo de Evaluación y Guías de Audiencias de alumnos, } \\
\text { profesores y coordinadores }\end{array}$ \\
\hline FUENTES & $\begin{array}{l}\text { Alumnos }(\mathbf{A}) \\
\text { Profesores }(\mathbf{P}) \\
\text { Coordinadores (C) } \\
\text { Comité Interno (CI) } \\
\text { Comité Externo (CE) }\end{array}$ \\
\hline INSTRUMENTOS & $\begin{array}{l}\text { Escala de valoración } \\
\text { Cuestionarios } \\
\text { Autoinformes } \\
\text { Guías de Entrevistas }\end{array}$ \\
\hline
\end{tabular}

De esta manera, se establece el diseño de un sistema de recogida de información cruzada, sobre las distintas variables trabajadas en el proyecto (entrada, proceso y producto) asumibles desde diversas finalidades como eficacia, eficiencia y funcionalidad, a partir de las cuales, en su conjunto, se refleja la calidad del plan de evaluación. Por otro lado, nos encontramos con la información aporta- da por cada fuente a través de los distintos instrumentos, mostrando un esquema de información donde se añade la aportada por el Comité Externo, ya que este actuaba como elemento de validación de la información aportada por el Comité Interno - ver tabla 10 de ejemplo-: 
Ramos, G. (2005). Elementos para el diseño de planes de evaluación de programas de teleformación en la empresa. RELIEVE: v. 11, n. 2, p. 3-23. http://www.uv.es/RELIEVE/v11n2/RELIEVEv11n2_5.htm

TABLA 10.- Esquema de información cruzada Triangulación de información, de fuentes e instrumentos en las diferentes dimensiones y variables (tabla ejemplo).

\begin{tabular}{|c|c|c|c|c|c|c|c|}
\hline & & FUENTE & ALUMNO & PROFESOR & COORDIN. & $\begin{array}{l}\text { COMITÉ } \\
\text { INTERNO }\end{array}$ \\
\hline & & & INSTRUMENTO & \multirow{2}{*}{$\begin{array}{l}\text { Cuestionario } \\
\text { y Audiencia }\end{array}$} & \multirow{2}{*}{$\begin{array}{l}\text { Autoinforme } \\
\text { y Audiencia }\end{array}$} & \multirow{2}{*}{$\begin{array}{l}\text { Cuestionario } \\
\text { y Audiencia }\end{array}$} & \multirow{2}{*}{$\begin{array}{l}\text { Escala de } \\
\text { Valoración }\end{array}$} \\
\hline \multirow{15}{*}{ 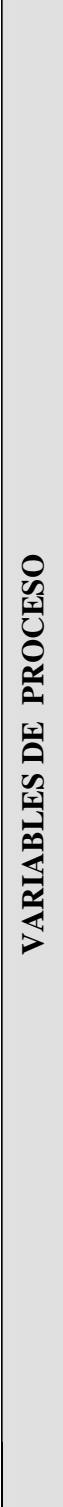 } & DIMENS & Criterio & UNIDADES DE INFORMACIÓN & & & & \\
\hline & \multirow{10}{*}{ 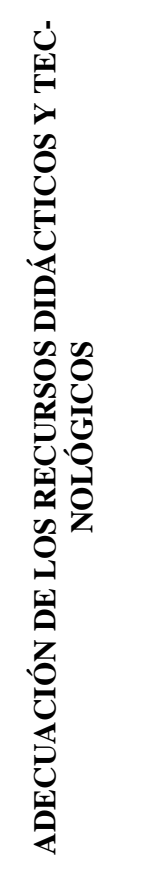 } & \multirow{10}{*}{ 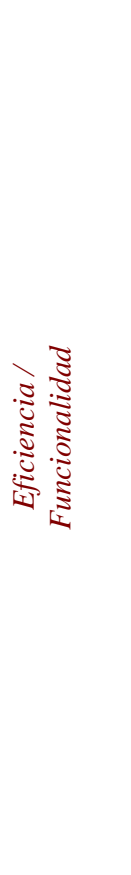 } & Es adecuada la Videoconferencia & Item 14 & $X$ & Item 9 & \\
\hline & & & Es adecuada la Audioconferencia & Item 15 & $X$ & Item 9 & \\
\hline & & & Es adecuado el uso de Internet & Item 16 & $X$ & & \\
\hline & & & Es adecuado el Chat & Item 17 & $X$ & Item 9 & \\
\hline & & & $\begin{array}{l}\text { Es adecuado el uso del Correo Elec- } \\
\text { trónico }\end{array}$ & & $X$ & Item 9 & \\
\hline & & & $\begin{array}{l}\text { Es adecuado el uso de la Comparti- } \\
\text { ción de aplicaciones }\end{array}$ & Item 18 & $X$ & Item 9 & \\
\hline & & & Es adecuado el Proyector de opacos & Item 19 & $X$ & Item 9 & \\
\hline & & & $\begin{array}{l}\text { Mejor recurso para este tipo de } \\
\text { acción formativa }\end{array}$ & & $X$ & Item 9 & \\
\hline & & & $\begin{array}{l}\text { Adecuación del manejo de la cámara } \\
\text { de vídeo }\end{array}$ & & $X$ & & \\
\hline & & & COMITÉ EXTERNO & $\boldsymbol{X}$ & $\boldsymbol{X}$ & & \\
\hline & \multirow{4}{*}{ 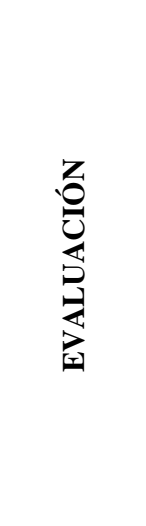 } & \multirow{4}{*}{ 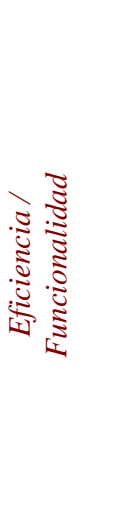 } & $\begin{array}{l}\text { Los procedimientos de evaluación } \\
\text { son variados y adecuados a las } \\
\text { competencias que se desea obtener } \\
\text { en los alumnos }\end{array}$ & & & & Item 130 \\
\hline & & & $\begin{array}{l}\text { El alumno es informado personal- } \\
\text { mente de sus resultados }\end{array}$ & & & & Item 138 \\
\hline & & & $\begin{array}{l}\text { El profesor decide alternativas de } \\
\text { itinerario individual para cada } \\
\text { alumno teniendo en cuenta los } \\
\text { resultados de la Evaluación }\end{array}$ & & & & Item 137 \\
\hline & & & COMITÉ EXTERNO ${ }^{[12]}$ & & $\boldsymbol{X}$ & & $\boldsymbol{X}$ \\
\hline
\end{tabular}

Para finalizar el proceso de validación se aporta un análisis lógico de congruencias y discrepancias de las valoraciones dadas por cada una de las fuentes sobre los puntos fuertes, débiles del sistema evaluado y sobre los aspectos a mejorar del mismo, es decir, sobre las conclusiones/ toma de decisiones aporta- das en cada informe de evaluación - ver tabla 11 de ejemplo-, analizándose a partir de ello, el grado de concordancia existente a través de un análisis cualitativo, el cual atiende a las dimensiones de evaluación y a la coincidencia entre fuentes. 


\begin{tabular}{|c|c|c|c|c|c|c|c|c|c|c|c|c|c|c|c|c|c|}
\hline \multicolumn{18}{|c|}{ Visor del Profesor/Tutor } \\
\hline \multirow{2}{*}{$\begin{array}{c}\text { PUNTOS } \\
\text { FUERTES }\end{array}$} & \multicolumn{5}{|c|}{ FUENTES } & \multirow{2}{*}{$\begin{array}{l}\text { PUNTOS } \\
\text { DÉBILES }\end{array}$} & \multicolumn{5}{|c|}{ FUENTES } & \multirow{2}{*}{$\begin{array}{l}\text { ASPECTOS A } \\
\text { MEJORAR }\end{array}$} & \multicolumn{5}{|c|}{ FUENTES } \\
\hline & A & $\mathbf{P}$ & C & CI & CE & & A & $\mathbf{P}$ & C & CI & CE & & $\mathbf{A}$ & $\mathbf{P}$ & C & CI & CE \\
\hline $\begin{array}{l}\text { La Comodidad } \\
\text { del Visor }\end{array}$ & & $\mathbf{X}$ & & & & $\begin{array}{l}\text { Introduce } \\
\text { demasiados } \\
\text { recursos en } \\
\text { pantalla }\end{array}$ & & & & $\mathbf{X}$ & & $\begin{array}{l}\text { Se debería reducir } \\
\text { el espacio destinado } \\
\text { al Chat }\end{array}$ & & $\mathbf{X}$ & $\mathbf{X}$ & $\mathbf{X}$ & \\
\hline $\begin{array}{l}\text { La distribución } \\
\text { de la pantalla es } \\
\text { clara y organiza- } \\
\text { da }\end{array}$ & & $\mathbf{X}$ & & $\mathbf{X}$ & & $\begin{array}{l}\text { Demasiados } \\
\text { elementos } \\
\text { activos en la } \\
\text { pantalla }\end{array}$ & & & & $\mathbf{X}$ & & $\begin{array}{l}\text { Incrementar los } \\
\text { recursos comple- } \\
\text { mentarios y el } \\
\text { acceso a ellos }\end{array}$ & & & & $\mathbf{X}$ & \\
\hline $\begin{array}{l}\text { Facilidad en el } \\
\text { acceso y comu- } \\
\text { nicación con los } \\
\text { alumnos }\end{array}$ & & $\mathbf{X}$ & & $\mathbf{X}$ & $\mathbf{X}$ & $\begin{array}{l}\text { No es posible } \\
\text { salirse de la } \\
\text { organización } \\
\text { de contenidos }\end{array}$ & & & & $\mathbf{X}$ & & $\begin{array}{l}\text { Mayores posibili- } \\
\text { dades de la audio- } \\
\text { conferencia }\end{array}$ & & $\mathbf{X}$ & & & \\
\hline $\begin{array}{l}\text { Las actividades } \\
\text { que se realizan } \\
\text { en el Visor y que } \\
\text { se llevan a cabo } \\
\text { con aplicaciones } \\
\text { compartidas }\end{array}$ & & & & $\mathbf{X}$ & $\mathbf{X}$ & $\begin{array}{l}\text { Demasiado } \\
\text { control del } \\
\text { tiempo }\end{array}$ & & & & $\mathbf{X}$ & & & & & & & \\
\hline $\begin{array}{l}\text { El seguimiento } \\
\text { continuo, ya que } \\
\text { permite controlar } \\
\text { lo que hace cada } \\
\text { alumno. Control } \\
\text { directo }\end{array}$ & & $\mathbf{X}$ & & $\mathbf{X}$ & & $\begin{array}{l}\text { El acceso a los } \\
\text { diferentes } \\
\text { componentes } \\
\text { del programa } \\
\text { no es tan ágil } \\
\text { como se pre- } \\
\text { tende }\end{array}$ & & $\mathbf{X}$ & & & & & & & & & \\
\hline $\begin{array}{l}\text { Incluye todos los } \\
\text { aspectos que se } \\
\text { necesita }\end{array}$ & & $\mathbf{X}$ & & $\mathbf{X}$ & & $\begin{array}{l}\text { El desarrollo } \\
\text { de determina- } \\
\text { das actividades } \\
\text { puede verse } \\
\text { interferido por } \\
\text { problemas } \\
\text { técnicos }\end{array}$ & & $\mathbf{X}$ & & & & & & & & & \\
\hline $\begin{array}{l}\text { Su empleo no } \\
\text { requiere muchos } \\
\text { conocimientos } \\
\text { informáticos }\end{array}$ & & & & $\mathbf{X}$ & & $\begin{array}{l}\text { No se puede } \\
\text { controlar la } \\
\text { eficacia del } \\
\text { trabajo del } \\
\text { alumno }\end{array}$ & & & & & $\mathbf{X}$ & & & & & & \\
\hline
\end{tabular}

(tabla ejemplo)

De todo ello y como producto final creemos haber aportado en este segundo estudio una estructura global de planificación y desarrollo de un proceso de evaluación de programas de teleformación (Objetivo Análisis I) y de un proceso de valoración de la validez de la definición de indicadores a partir de las conclusiones de diversas fuentes de información en dicho proceso de evaluación (Objetivo Análisis II), aunque en este artículo se haya presentado de una manera muy sucinta debido al formato del mismo.

\section{CONCLUSIONES GENERALES}

A partir de la investigación desarrollada podemos concluir de forma muy general, $\mathrm{y}$ atendiendo a los resultados obtenidos en ambos estudios, que: 
-La elección de los recursos tecnológicos para el diseño y desarrollo de los programas de formación constituye uno de los puntos clave en un proyecto de teleformación.

- Se han de considerar a los recursos tecnológicos como criterios internos al concepto de teleformabilidad de un programa de formación.

- Se han de identificar adecuadamente el rol que juega cada uno de los recursos en el proceso de formación, su aplicación y su repercusión formativa.

- Los indicadores para la evaluación de los programas de teleformación han de girar alrededor de todos los elementos que imponen los contenidos de formación, de la implementación del programa y de las características específicas del medio.

- Asimismo, se han de establecer estándares de evaluación para los distintos contenidos formativos teniendo en cuenta indicadores como el recurso tecnológico utilizado y su aprovechamiento metodológico.

- Se hace necesario un modelo integrador de lo tecnológico con lo pedagógico para la evaluación de la teleformación. Que globalice de forma adecuada todos los requerimientos $\mathrm{y}$ necesidades que se plantean en la evaluación de este tipo de programas de formación. En esta línea, hemos propuesto la aplicación del modelo de De la Orden (1988, 1990, 1992, 1997), por su carácter sistémico y por que permite identificar de forma clara los diversos componentes de un programa de formación en este caso de teleformación- con sus posibles efectos para orientar el análisis de Planes. Es un modelo de calidad que intenta superar la consideración aislada de las características específicas de los distintos componentes (contexto, entrada, proceso, producto) y tratar de especificar los rasgos comunes a todas ellas. Permite además elaborar indicadores para evaluar la eficacia, eficiencia y funcionalidad de un programa con los diferentes aspectos a tener en cuenta, hecho que le lleva a delimitar muy bien la finalidad de la evaluación.
- El Protocolo de Actuación para Evaluadores que aquí proponemos - ver Anexo- se convierte en una herramienta para abordar el diseño y desarrollo de Planes de Evaluación en este ámbito planteada desde criterios de calidad, tanto a nivel político (orientando el diseño y desarrollo de programas al permitir un análisis adecuado de soluciones para diversos sectores profesionales), pasando por las PY$\mathrm{ME}$ (puesto que puede orientarles en el análisis y la toma de decisiones acerca de la adecuación de las soluciones tecnológicas según su sector profesional), hasta los proveedores de teleformación (como ayuda para el análisis de programas de formación y directrices para el establecimiento de inversiones) (Consecución del objetivo Estudio I).

En conjunto con los dos estudios empíricos se ha intentado comprobar a partir del desarrollo de un conjunto de experiencias la viabilidad de un sistema de evaluación y/o la necesidad de la creación de un plan de evaluación para programas de teleformación, identificando indicadores relevantes que se han de tener en cuenta a la hora de evaluar el diseño de este tipo de programas de formación.

Todo ello siendo conscientes de que:

- Los resultados obtenidos están ligados a los recursos tecnológicos que fueron utilizados ${ }^{[13]}$, por lo que, se precisa una revisión continua de los recursos tecnológicos que actualmente están en uso como mejora y ampliación de los resultados.

- El valor global de esta investigación reside en que se sitúa en la base de la construcción del conocimiento evaluativo para este ámbito.

\section{REFERENCIAS BIBLIOGRÁFICAS}

De la Orden, A. (1988). La calidad de la educación. Bordón, 42, 2, 149-161.

De la Orden, A. (1990). Evaluación de los efectos de los programas de intervención. Revista de investigación educativa, 8, 16, 6176.

De la Orden, A. (1992). Calidad y Evaluación de la Enseñanza Universitaria. Actas del 
Congreso Internacional de Universidades. Madrid: Universidad Complutense.

De la Orden, A.; Asensio, I.; Carballo, R.; Fernández Díaz, J.; Fuentes, A.; García Ramos, J. M.; y Guardia, S. (1997). Desarrollo y validación de un modelo de calidad universitaria como base para su evaluación. RELIEVE, 3, $\quad 1 . \quad$ Consultado en http://www.uv.es/relieve/v3n1/RELIEVEv3n 1_2.htm el 10 de Septiembre de 2005.

Jiménez, B. (Coord.) (1999). La evaluación de programas, centros y profesores. Madrid: Editorial Síntesis, S.A.

Jornet, J. M.; Suárez, J. M. y Belloch, C. (1998). Metodología de evaluación de programas de formación profesional, ocupacional y continua. Leonardo project: Eurodialogue Evaluation. Valencia: Fundació Universitat-Empresa de València (ADEIT).

Jornet, J. M.; Suárez, J. M. y Perales, M. J (2000). La evaluación de la formación ocu- pacional y continua. Revista de Investigación Educativa, 18 (2), 521-538.

Jornet, J. M.; Suárez, J. M. y Pérez Carbonell, A. (2000). La validez en la evaluación de programas. Revista de Investigación Educativa, 18 (2), 341-356.

Martínez Mut, B. (2003). La formació a l'empresa. Enginyeria dels processos Educatius. Valencia: Editorial UPV.

Merrill, M. D. y Twitchell D. G (1994). Instructional design theory. Englewood Cliffs (NJ): Educational Tecnology Publications.

Perales, M. J. (2000). Enfoques de evaluación de la formación ocupacional y continua. Estudio de validación de un modelo. Valencia: Tesis doctoral. Universitat de València.

Swanborn, P. G. (1996). A common base for quality control criteria in quantitative and qualitative research. Quality and quantity, 30, 19-35.

\section{NOTAS}

[1] Esta experiencia de evaluación es el denominado Proyecto TETRA (Telematics Training): proyecto de desarrollo y evaluación de programas de formación en las PYME mediante teleformación. La evaluación del proyecto integró dos vertientes: la evaluación de materiales y la evaluación de programas.

[2] Concretamente por el Grupo de Evaluación y Medición (GEM) y la Unidad de Tecnología Educativa (UTE).

[3] Estas iniciativas pretendían contribuir a la adaptación de los trabajadores a las transformaciones industriales y tecnológicas y mejorar el funcionamiento del mercado de trabajo ayudando a los trabajadores a adaptarse a las exigencias de la Nueva Sociedad de la Información.

[4] Cada programa de formación hace referencia a una ocupación profesional. En cuanto a su estructura, las ocupaciones se enmarcaron en familias profesionales.

[5] Administración y Oficinas, Artesanía, Cerámica, Comercio, Idiomas, Industrias Gráficas, Industria Textil, Informática, Manufacturas diversas, Montaje e Instalación, Seguros y Finanzas, Servicios a la Comunidad y Personales, Servicios a las Empresas, Técnicas Empresariales, Textil y Turismo y Hostelería.

[6] (0) No Teleformable

(1) Teleformable/ No necesario: cuando las condiciones concretas permitían la teleformabilidad pero esta se desaconsejaba, por diferentes razones de estructura formativa.

(2) Teleformable/ Necesario, Conveniencia Baja: cuando las posibilidades de teleformación eran reducidas bien por su aplicabilidad - atendiendo a las diferentes herramientas tecnológicas para la teleformación- o por razones de estructura formativa.

(3) Teleformable/Necesario, Conveniencia Media: cuando las posibilidades de teleformación eran sustanciales, teniendo en cuenta las diferentes herramientas tecnológicas para la teleformación y las características de la estructura formativa avalaban su aplicabilidad en una proporción sustancial del programa formativo o de los módulos específicos en cuestión.

(4) Teleformable/Necesario, Conveniencia Alta: cuando las posibilidades de teleformación eran muy amplias, tomando en cuenta las diferentes herramientas tecnológicas para la teleformación y las características de la estructura formativa avalaban su aplicabilidad en una proporción muy elevada del programa formativo o de los módulos específicos en cuestión. 
[7] A partir de los siguientes niveles de clasificación de concordancia: 0-0.20: Muy baja; 0.21-0.40: Baja; 0.41-0.60: Media; 0.61-0.80: Alta; 0.81-1: Muy Alta.

[8] Las definiciones de los criterios de calidad que nos han servido de base en todo el estudio son las establecidas por De la Orden en diferentes ocasiones (1988; 1990; 1992; 1997).

[9] Esta propuesta, es inicial y tentativa y debería ser probada en otros marcos, en el que se pudiese valorar la homogeneidad de utilización de los estándares a través de diferentes situaciones y por diversos evaluadores como base de fiabilidad y validez del Protocolo.

[10] Escala de Interpretación del estándar de referencia: 0-0.20: Nada importante; 0.21-0.40: Poco importante; 0.41-0.60: Importante; 0.61-0.80: Muy importante; 0.81-1: Fundamental.

[11] En estas tablas resúmenes aparecen sólo coloreados los Estándares de Referencia descritos a continuación:

Fundamental (0.81-1) representado por el color Violeta

Muy Importante (0.61-0.80) representado por el color Azul Claro

Importante (0.41-0.60) representado por el color Verde

Cuando la casilla no está representada con ningún color es debido a que el Estándar es poco o nada importante.

[12] Valoración general extraída a partir de la simulación directa sobre el desarrollo de un Programa de Formación, en audiencias con Profesores y de la revisión del Informe del Comité Interno.

[13] Páginas HTML, Otros tipos de Páginas (Java Perl o Script), Correo Electrónico, Grupos de Discusión, Recursos Cliente/ Servidor, Chat y Videoconferencia.

\section{ANEXO: Protocolo de Evaluación}

\section{Presentación}

La Guía de Actuación para la Aplicación de Estándares es un instrumento que pretende recoger la información necesaria para que el Evaluador/a analice la eficiencia/ funcionalidad del Programa de Formación teniendo en cuenta la Tecnología y la Metodología Didáctica que ella nos permite desarrollar en el Programa. Todo ello en función del contenido del mismo.

Es decir, esta Guía, nos permite describir, a la vista del Programa de Formación y del desarrollo metodológico del mismo, los Estándares para poder evaluar si el diseño del Programa de Formación es adecuado o no. Por este motivo en esta guía pretendemos recoger información de los contenidos, de los implicados y de los recursos tecnológicos utilizados.

Para ello se hace necesario:

1.- Identificar el tipo de Programa de Formación según su contenido.

2.-Desarrollar Audiencias o Entrevistas con los implicados en el Diseño e Implementación del Programa de Formación.

3.-Identificar el funcionamiento de los Estándares en el desarrollo del Programa de Formación.

- Identificar los Elementos Tecnológicos diseñados en el Programa de Formación.

- Identificación del USO de los Estándares

Forma de Aplicación (Ver apartados siguientes)

1.- IDENTIFICAR EL TIPO DE PROGRAMA DE FORMACIÓN SEGÚN SU CONTENIDO

1. 1.- Título del Programa de Formación:

1. 2.- Familia Profesional o sector profesional al que pertenece

Recomendación: 
Identifique la Familia Profesional a la cual pertenece según la temática del programa; si no se halla el mismo programa, es recomendable utilizar el criterio de similitud de contenido. Intente, entonces, ubicarlo en la Familia Profesional más cercana a su contenido y tipo de competencias a desarrollar.

Si su clasificación no es clara, tome como referencia las Familias Profesionales más similares (por ejemplo, si se tratara de un programa de Comercio Exterior, tome Administración y Oficinas, Servicios a la Empresas, Técnicas empresariales...). Ver Anexo I. Listado referencial de Programas de Formación trabajados en nuestro estudio y posteriormente especificar el nombre del Programa en la opción de "Otras".

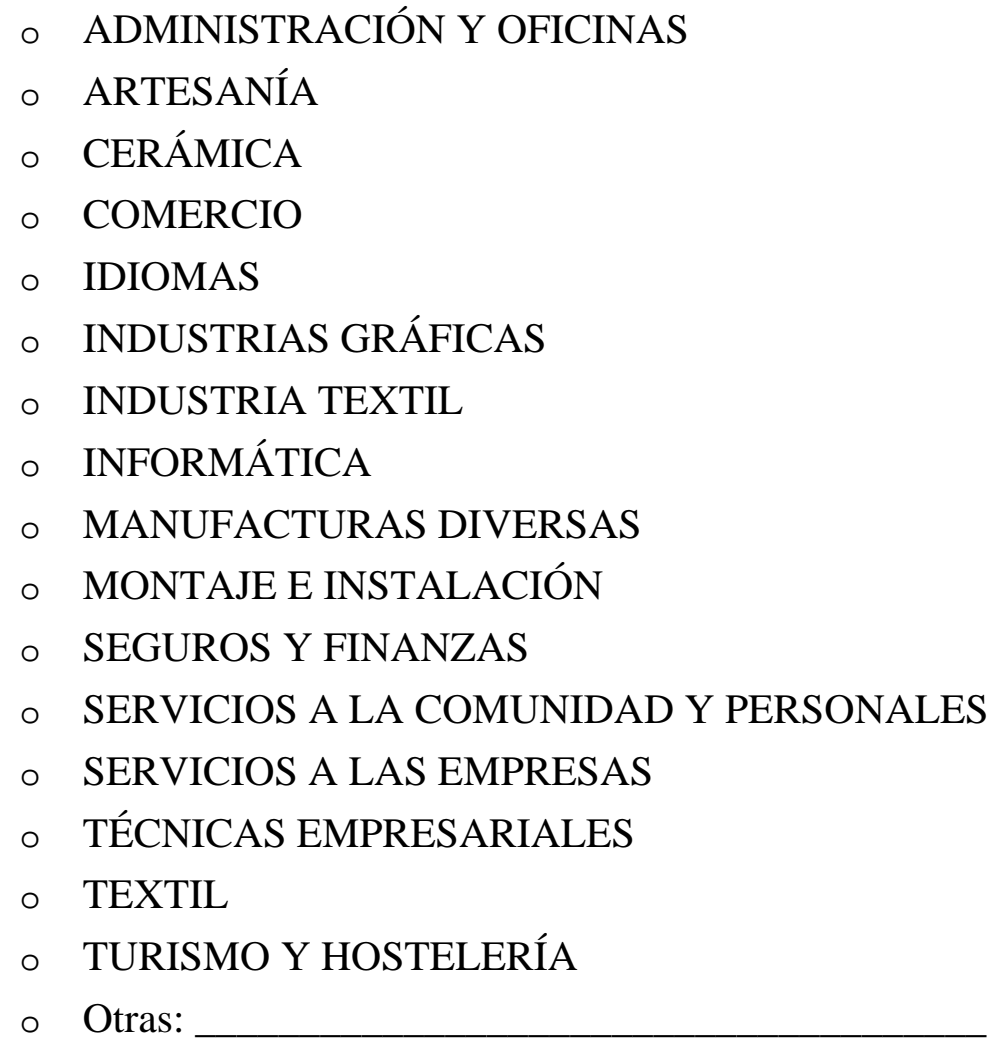

\section{3.- Tipo de Contenidos}

- Módulo Teórico. Número de Horas

- Módulo Práctico. Número de Horas

- Número de Horas Totales del Programa de Formación

\section{Recomendación:}

Teniendo en cuenta el número de horas totales del curso calcular la proporción de forma ponderada, de las horas Teóricas y la proporción de horas Prácticas que se establecen en el Programa de Formación.

- Proporción de horas Teóricas

- Proporción de horas Prácticas

\section{4.- Nivel de teleformabilidad de los Contenidos del Programa de Formación}

\section{Recomendación:}

Una vez realizados los cálculos proporcionales de horas de dedicación a los Módulos, (ver Anexo II. Tablas relación existente entre la proporción de horas dedicadas a la Teoría y a la Práctica en los 
distintos Programas de Formación analizados y el nivel de teleformabilidad que alcanzan dichos contenido), anotar a continuación el grado de teleformabilidad del Programa, según los datos descrito en el Estudio Empírico.

- Grado o Nivel de teleformabilidad Global del Programa o del Programa con contenido similar

- Grado o Nivel de teleformabilidad Teórico del Programa o del Programa con contenido similar

- Grado o Nivel de teleformabilidad Práctico del Programa o del Programa con contenido similar

\section{2.- DESARROLLAR ENTREVISTAS CON LOS IMPLICADOS EN EL PROGRAMA DE FOR- MACIÓN}

El Objetivo de este apartado del proceso de Evaluación es conocer a partir de una/s Entrevista/s, programada y estructurada, con cada uno de los implicados en el diseño y desarrollo del Programa de Formación, cómo se han diseñado y aplicado los distintos Estándares en estos contenidos y cuál ha sido la funcionalidad didáctica de los mismos.

Para ello es conveniente realizar Entrevista/s con:

- Los Técnicos en informática y telemática que han diseñado e implementado los distintos recursos tecnológicos en el Programa de Formación.

- Los Gestores de Formación, con el fin de establecer, según su experiencia, la relevancia/ adecuación/ funcionalidad de los distintos elementos tecnológicos en la organización y gestión del proceso de formación.

- El/los Profesor/es y Tutor, para conocer la utilización y funcionalidad de los distintos recursos tecnológicos en el proceso de enseñanza- aprendizaje. Visionar el Visor del Profesor/Tutor.

- Los Alumnos, los cuales nos informarán de la adecuación de los recursos tecnológicos y de su importancia/ funcionalidad en el proceso de aprendizaje. Visionar el Visor del Alumno.

\section{Recomendación:}

El cómo y el cuándo de cada una de las Entrevistas vendrá determinado por las características de las Audiencias a la que va dirigida y los requisitos de la misma.

\section{3.- IDENTIFICAR EL FUNCIONAMIENTO DE LOS ESTÁNDARES EN EL DESARROLLO DEL PROGRAMA DE FORMACIÓN}

El objetivo de este apartado del proceso de Evaluación es identificar los elementos tecnológicos diseñados para el Programa de Formación e identificar el uso de los mismos en el desarrollo de los Programas.

Por lo que, para identificar el uso de los recursos tecnológicos hemos de evaluar la adecuación del uso de cada recurso en el Programa de Formación que estamos evaluando, así como establecer la importancia de los mismos según el estándar de referencia obtenido en el estudio empírico de referencia ver apartado 3.1 y 3.2.-

\section{1.- Identificación del USO de los Estándares}

3.1.1. En primer lugar, se hace necesario identificar los Recursos Tecnológicos que se tuvieron en cuenta en el diseño del Programa de Formación para el desarrollo del proceso de enseñanza- aprendizaje. Por lo que, primeramente hemos de señalar en la tabla 1 que recurso no se ha utilizado con un aspa $(X)$ en la columna No lo utiliza.

3.1.2. En segundo lugar, el Evaluador/a ha de valorar el nivel de adecuación/utilización de los distintos Recursos Tecnológicos utilizados, previamente diseñados para el funcionamiento del Programa, en el proceso de enseñanza- aprendizaje.

La escala de valoración será la siguiente:

1 Muy Mal Utilizado

2 Bastante Mal Utilizado 
3 Utilizado de forma adecuada

4 Bastante Bien Utilizado

5 Muy Bien Utilizado

La puntuación más alta en la escala indica que el Recurso Tecnológico valorado propicia la metodología didáctica de la que parte en su grado máximo. Ver Anexo III. Metodología Didáctica de los Recursos Tecnológicos.

Se ha de diferenciar dicha utilización teniendo en cuenta los módulos (teoría/ práctica) - rellenar tabla 1- (PUNTUACIÓN A).

\begin{tabular}{|c|c|c|c|c|c|c|c|c|c|c|c|c|}
\hline & & & dec & Ciol & e u & del & curs & & & & & \\
\hline TITULO DEL PROGH & MA & E FC & MAC & & & & & & & & & \\
\hline $\begin{array}{c}\text { RECURSO } \\
\text { TECNOLÓGICO }\end{array}$ & & & & & & & $\begin{array}{l}\text { Utili } \\
\mathbf{f} \\
\text { ad }\end{array}$ & & $\begin{array}{r}\text { Basta } \\
\text { Uti }\end{array}$ & $\begin{array}{l}\text { Bien } \\
\text { do }\end{array}$ & & \\
\hline & $\mathbf{T}$ & $\mathbf{P}$ & $\mathbf{T}$ & $\mathbf{P}$ & $\mathbf{T}$ & $\mathbf{P}$ & $\mathbf{T}$ & $\mathbf{P}$ & $\mathbf{T}$ & $\mathbf{P}$ & $\mathbf{T}$ & $\mathbf{P}$ \\
\hline Páginas HTML & & & & & & & & & & & & \\
\hline Otras Páginas & & & & & & & & & & & & \\
\hline Correo Electrónico & & & & & & & & & & & & \\
\hline Grupos de discusión & & & & & & & & & & & & \\
\hline Cliente/Servidor & & & & & & & & & & & & \\
\hline Chat & & & & & & & & & & & & \\
\hline Videoconferencia & & & & & & & & & & & & \\
\hline
\end{tabular}

3.1.3. Teniendo en cuenta la adecuación de la utilización de los Recursos Tecnológicos en el Programa de Formación y el contenido del mismo, se ha de recoger la información sobre los Estándares de Referencia Teóricos y Prácticos obtenidos en el Estudio Empírico para el Programa de Formación aquí evaluado. Obteniéndose un perfil de referencia ideal. Datos que se pueden obtener en el Anexo IV. Tablas de Estándares de Referencia hallados en este estudio según los distintos Contenidos.

Posteriormente rellenar tabla 2 (PUNTUACIÓN B). 


\section{PUNTUACIÓN B \\ Importancia del recurso según el Programa de Formación a evaluar}

Titulo del Programa de Formación:

Familia Profesional o tipo de contenido al que pertenece:

\begin{tabular}{|l|l|l||}
\hline \multicolumn{1}{|c|}{ RECURSO TECNOLÓGICO } & $\begin{array}{r}\text { CRITERIO DE REFEREN } \\
\text { MÓDULOS TEÓRICOS }\end{array}$ & $\begin{array}{c}\text { CRITERIO DE REFERENC } \\
\text { MÓDULOS PRÁCTICOS }\end{array}$ \\
\hline Páginas HTML & & \\
\hline Otras Páginas (Java, Perl o Script) & & \\
\hline Correo Electrónico & & \\
\hline Grupos de discusión & & \\
\hline Cliente/Servidor & & \\
\hline Chat & & \\
\hline Videoconferencia & & \\
\hline \multicolumn{2}{|c|}{ Tabla 2. Criterios de Referencia de los recursos tecnológicos en este Programa de Formación } \\
\hline
\end{tabular}

3.1.4.- A partir de todos estos criterios de valoración se ha de proceder a establecer la Puntuación de Síntesis - ver tabla 3-, calculando los índices e interpretando los resultados - ver apartado 4 -

\begin{tabular}{|c|c|c|c|c|c|c|c|}
\hline \multicolumn{8}{|c|}{ PUNTUACIÓN DE SÍNTESIS } \\
\hline \multicolumn{8}{|c|}{$\begin{array}{l}\text { Titulo del Programa de Formación: } \\
\text { Familia Profesional o tipo de contenido al que pertenece: }\end{array}$} \\
\hline \multicolumn{4}{|c|}{ MÓDULOS TEÓRICOS } & \multicolumn{4}{|c|}{ MÓDULOS PRÁCTICOS } \\
\hline RECURSO TECNOLÓGICO & A & B & AxB & RECURSO TECNOLÓGICC & A & B & AxB \\
\hline Páginas HTML & & & & Páginas HTML & & & \\
\hline Otras Páginas & & & & Otras Páginas & & & \\
\hline Correo Electrónico & & & & Correo Electrónico & & & \\
\hline Grupos de discusión & & & & Grupos de discusión & & & \\
\hline Cliente/Servidor & & & & Cliente/Servidor & & & \\
\hline Chat & & & & Chat & & & \\
\hline Videoconferencia & & & & Videoconferencia & & & \\
\hline \multirow[b]{2}{*}{ Sumatorios } & $\mathrm{S}_{\mathrm{A}}$ & $\mathrm{S}_{\mathrm{B}}$ & $\mathrm{S}_{\mathrm{AB}}$ & \multirow[b]{2}{*}{ Sumatorios } & $\mathbf{S}_{\mathbf{A}}$ & $\mathrm{S}_{\mathrm{B}}$ & $\mathrm{S}_{\mathrm{AB}}$ \\
\hline & & & & & & & \\
\hline \multirow[b]{2}{*}{ Ratios ( $r$ ) } & $\mathrm{S}_{\mathrm{A}} / \mathbf{N}$ & $\mathbf{S}_{\mathbf{B}} / \mathbf{N}$ & $\mathbf{S}_{\mathrm{AB}} / \mathbf{N}$ & \multirow[b]{2}{*}{ Ratios ( $r$ ) } & $\mathrm{S}_{\mathrm{A}} / \mathbf{N}$ & $\mathrm{S}_{\mathrm{B}} / \mathrm{N}$ & $\mathbf{S}_{\mathrm{AB}} / \mathbf{N}$ \\
\hline & & & & & & & \\
\hline$r / m a ́ x$ & & & & $r / m a ́ x$ & & & \\
\hline \multicolumn{8}{|c|}{$\begin{array}{l}\text { Tabla 3. Puntuación síntesis de la adecuación /utilización del uso del recuso y la importancia d } \\
\text { mismo en el Programa de Formación a evaluar }\end{array}$} \\
\hline
\end{tabular}




\section{Interpretación de las puntuaciones}

Puede estructurarse atendiendo a las siguientes consideraciones:

- Puntuaciones A informan acerca de la adecuación en el uso de cada recurso. Por lo que aportan un perfil de adecuación tecnológica - información extraída de tabla 1-.

- Puntuaciones B, informan acerca de la importancia del mismo en el diseño del programa, atendiendo a los usos didácticos que pueden requerirse según el contenido y competencia a desarrollar. Así, aportan el perfil de referencia ideal acerca de la funcionalidad de cada recurso tecnológico en el programa evaluado - información extraída de tabla 2-.

- Las puntuaciones $\mathbf{A x B}$, aportan la información de síntesis acerca de la adecuación relativa teniendo en cuenta la funcionalidad del recurso. Se establece una puntuación única tomando como referencia el Estándar teórico particular de cada Programa de Formación (B) combinado con la utilización del recurso de cada uno de ellos (A).

- Los sumatorios y ratios, aportan una información global de cada aspecto, pudiéndose considerar escalables e interpretables según la escala teórica utilizada para la valoración.

- Además el evaluador/a obtiene un perfil diferencial de cada uno de los recursos Tecnológicos en estos contenidos, si comparamos la Columna A con la Columna $\mathbf{B}$, ya que informa de las discrepancias existentes respecto al Estándar de Referencia.

- La ratio dividido el máximo teórico en la columna A (r/máx) representa la puntuación obtenida en la ratio dividida por la puntuación máxima que pueden llegar a obtener cada recurso (5) en las valoraciones dadas. El resultado, en una escala de proporción (de 0 a 1), nos aporta información global de la adecuación tecnológica de los recursos interpretable según la siguiente escala teórica:

0-0.20: No adecuado
0.21-0.40: Adecuación medio baja
0.41-0.60: Adecuación media
0.61-0.80: Adecuación medio alta
0.81-1: Adecuación alta

- La ratio dividido el máximo teórico en la columna AxB (r/máx) representa la puntuación obtenida en la ratio dividida por la puntuación máxima que pueden llegar a obtener cada recurso

(5) en las valoraciones dadas, entendiendo que el máximo teórico representa al sumatorio de todos las puntuaciones B x 5, es decir, $A x B=\sum(5 x \underset{n=1}{B})$

. El resultado, en una escala de proporción (de 0 a 1), nos aporta información global de la adecuación tecnológica de los recursos teniendo en cuenta la eficiencia/funcionalidad del mismo, interpretable según la siguiente escala teórica:
0-0.20: No adecuado
0.21-0.40: Adecuación medio baja
0.41-0.60: Adecuación media
0.61-0.80: Adecuación medio alta
0.81-1: Adecuación alta

- Anexo I.

Listado referencial de Programas de Formación analizados en estudio empírico.

- Anexo II.

Tablas relación existente entre la proporción de horas dedicadas a la Teoría y a la Práctica en los distintos Programas de Formación analizados y el nivel de teleformabilidad que alcanzan los contenidos.

- Anexo III.

Metodología Didáctica de los Recursos Tecnológicos.

\section{- Anexo IV.}

Tablas de Estándares de Referencia hallados según los distintos Contenidos. 
Ramos, G. (2005). Elementos para el diseño de planes de evaluación de programas de teleformación en la

empresa. RELIEVE: v. 11, n. 2, p. 3-23. http://www.uv.es/RELIEVE/v11n2/RELIEVEv11n2_5.htm

\title{
ABOUT THE AUTHORS / SOBRE LOS AUTORES
}

Genoveva Ramos (Genoveva.Ramos@uv.es). Doctora en Filosofía y Ciencias de la Educación. En la actualidad profesora asociada en el Dpto. de Métodos de Investigación y Diagnóstico en Educación de la Universitat de València (España). Desarrolla su labor docente e investigadora en el ámbito de la Medición y la Evaluación Educativa, concretamente dentro del Grupo de Evaluación y Medición -GEM-. Dicha labor se centra en: la evaluación de resultados escolares, en la evaluación de programas (en ámbitos como la Formación Ocupacional, Profesional y Continua, la Formación Continua en Sanidad y la teleformación) y en el desarrollo de indicadores de calidad tanto para Centros como para la evaluación de Sistemas Educativos

\section{ARTICLE RECORD / FICHA DEL ARTÍCULO}

\begin{tabular}{|c|c|}
\hline $\begin{array}{l}\text { Reference / } \\
\text { Referencia }\end{array}$ & $\begin{array}{l}\text { Ramos, Genoveva (2005). Elementos para el diseño de planes de evaluación de programas de teleforma- } \\
\text { ción en la empresa. Revista ELectrónica de Investigación y EValuación Educativa, v. 11, n. } 2 \text {. } \\
\text { http://www.uv.es/RELIEVE/v11n2/RELIEVEv11n2_5.htmConsultado en (poner fecha). }\end{array}$ \\
\hline Title / Título & $\begin{array}{l}\text { Elementos para el diseño de planes de evaluación de programas de teleformación en la empresa. [Ele- } \\
\text { ments for evaluation plans design about e-learning programs in the company area] }\end{array}$ \\
\hline Authors / Autores & Genoveva Ramos \\
\hline $\begin{array}{l}\text { Review / } \\
\text { Revista }\end{array}$ & Revista ELectrónica de Investigación y EValuación Educativa (RELIEVE), v. 11, n. 2 \\
\hline $\begin{array}{l}\text { Publication date / } \\
\text { Fecha de publicación }\end{array}$ & $\begin{array}{l}2005 \text { (Reception Date: } 2005 \text { July 07; Approval Date: } 2005 \text { November 8; Publication Date: } 2005 \text { No- } \\
\text { vember 11) }\end{array}$ \\
\hline $\begin{array}{l}\text { Abstract / } \\
\text { Resumen }\end{array}$ & $\begin{array}{l}\text { From a broad research framework, this paper's objective is to reflect on different ways to develop E- } \\
\text { learning evaluation plans for companies whose employees are in continuous training. Two empirical } \\
\text { studies are presented that provide substantive references for identifying the standards, indicators and } \\
\text { variables that can be considered for this type of evaluation plan. The results of different kinds of analyses } \\
\text { have resulted in a guideline of standards usable in an Evaluation Protocol, and an outline to facilitate } \\
\text { the planning and development of these kinds of evaluations. } \\
\text { Desdeuna perspectiva amplia, este artículo tiene como objetivo aportarelementosde reflexión y algu- } \\
\text { nas soluciones en investigación evaluativa, a fin de abordary orientar los planes de evaluación de la tele- } \\
\text { formación en el ámbito de laformación continua en la empresa. Se presentan dos estudiosempíricos que } \\
\text { identifican estándares, indicadores y variables que puedan serconsiderados en este tipo de planes de eva- } \\
\text { luación. Se propone una estructura deestándares utilizable en el marco de un Protocolo de Evaluación. }\end{array}$ \\
\hline $\begin{array}{l}\text { Keywords } \\
\text { Descriptores }\end{array}$ & $\begin{array}{l}\text { E-learning, E-learning Evaluation Plans, E-learning Programs design. } \\
\text { Teleformación, Planes de Evaluación de Teleformación, Diseño de Programas de Teleformación }\end{array}$ \\
\hline $\begin{array}{l}\text { Institution / } \\
\text { Institución }\end{array}$ & Universidad de Valencia (España) \\
\hline $\begin{array}{l}\text { Publication site / } \\
\text { Dirección }\end{array}$ & http://www.uv.es/RELIEVE \\
\hline
\end{tabular}

[ ISSN: 1134-4032 ]

\begin{abstract}
(C) Copyright, RELIEVE. Reproduction and distribution of this articles it is authorized if the content is no modified and their origin is indicated (RELIEVE Journal, volume, number and electronic address of the document).

(C) Copyright, RELIEVE. Se autoriza la reproducción y distribución de este artículo siempre que no se modifique el contenido y se indique su origen (RELIEVE, volumen, número y dirección electrónica del documento).
\end{abstract}

